\section{Risk of VTE among users of oral contraceptives}

We have recently reviewed two studies, ${ }^{1}$ a cohort study conducted in Denmark, ${ }^{2}$ and a case-control study conducted in The Netherlands, ${ }^{3}$ in which it was claimed that the risk of venous thromboembolism (VTE) among users of oral contraceptives (OCs) containing desogestrel, gestodene, drospirenone and cyproterone is greater than among users of levonorgestrelcontaining OCs. We concluded that in both studies the comparisons among the progestogens were not valid due to methodological limitations.

The Danish study linked prescription data recorded in one national registry to hospital discharge diagnoses of VTE recorded in another registry. The investigators stated that in an earlier validation study $10 \%$ of the diagnoses documented between 1994 and 1998 "were uncertain". In the study under review they acknowledged that they relied on the "final discharge diagnoses as reported", and that they were unable to "evaluate the validity of each included diagnosis of [VTE]". ${ }^{2}$

Since publication of our review new information has come to light that bears on the validity of the registry-recorded diagnoses. In a cohort study that included 27178 men and 29876 women aged 50-64 years, Severinsen and her colleagues examined the medical records of 1100 cases of registry-recorded VTE. 4 The diagnosis was incorrect in $25 \%$ of cases diagnosed in hospital wards, and in $69 \%$ of cases diagnosed in emergency departments; the latter cases constituted $41 \%$ of the total. Incorrect diagnoses were more commonly recorded among women than among men. A stratified analysis did not show an impact of age on diagnostic precision.

It is difficult to reconcile the findings of Severinsen et al. with the assumption that the diagnosis was uncertain in about $10 \%$ of the cases of VTE, ${ }^{2}$ even though that estimate was made among women of fertile age. Based on the wording used by the authors it can be assumed that the VTE incidence rates among the compared OCs were based on all VTE diagnoses including VTE diagnosed in emergency departments. If so, Severinsen's results suggest that the diagnosis was not only uncertain, but in at least $40 \%$ of the cases it was wrong. If the analysis was based only on hospital ward cases, the diagnosis was incorrect in about $29 \%$ of the female patients

Relative to levonorgestrel the relative risks for the compared OCs were small $(<2)$, and the major diagnostic imprecision suggested by Severinsen's data would be sufficient to nullify the findings. It obliges Lidegaard to verify the diagnoses in his study.

Samuel Shapiro, FCP(SA), FRCP(E)

Visiting Professor of Epidemiology, Department of Epidemiology, University of Cape Town,

Cape Town, South Africa.

E-mail:samshap@mweb.co.za

Juergen Dinger, $\mathrm{MD}, \mathrm{PhD}$

Director, Berlin Center for Epidemiology and

Health Research, Berlin, Gemany.

E-mail:dinger@zeg-berlin.de

\section{Statements on funding and}

Competing interests The authors presently consult, and in the past have consulted, with manufacturers of products discussed in this letter.

\section{References}

Shapiro S, Dinger J. Risk of venous thromboembolism among users of oral contraceptives: a review of two recently published studies J Fam Plann Reprod Health Care 2010; 36: 33-38.

2 Lidegaard Ø, Løkkegaard E, Svendsen AL, Agger C. Hormonal contraception and risk of venous thromboembolism: national follow-up study. $B M J$ 2009; 339: b2890.

Van Hylckama Vlieg A, Helmerhorst FM, Effects of oestrogen dose and progestogen type on venous thrombotic risk associated with oral
contraceptives: results of the MEGA case-control study. BMJ 2009; 339: b2921.

4 Severinsen MT, Kristensen SR, Overvad K, Dethlefsen C, Tjønneland A, Johnsen SP. Venous thromboembolism discharge diagnoses in the Danish Nationa Patient Registry should be used with caution. J Clin Epidemiol 2010; 63: 223-228

\section{Critique of a Danish cohort study on} hormonal contraception and VTE

Thanks to Samuel Shapiro and Jürgen Dinger (S\&D) for their altruistic interest in and concern for possible bias and confounding in two recently published studies on use of oral contraceptives (OCs) and the risk of venous thromboembolism (VTE), as detailed in their review article ${ }^{1}$ in the January 2010 issue of the Journal of Family Planning and Reproductive Health Care. One of the two studies under discussion was a national Danish cohort study. ${ }^{2}$

The two authors' concern is to question whether bias and confounding could explain how different types of progestogens in OCs seem to play a differential role in the risk of VTE. However, S\&D don't stop with questioning. They actually conclude first that the results of both studies are invalid, and second that the best scientific evidence (taking all studies into account) is that the progestogen type in the combined OC has no influence on the risk of VTE.

These rather bombastic conclusions necessitate a validation of each of their points of concern for the Danish cohort study.

\section{Control for duration of use}

S\&D correctly state that the risk of VTE is highest during the first months of use. It is also correct that some (in fact few, however) shortterm users of OC with levonorgestrel (LNG) might have used the pill for a longer period (before our study window started in 1995), namely the small fraction of the LNG short-term users beginning their short use in the beginning of 1995. While this potential left censoring bias could influence users of OC with LNG more than users of OC with drospirenone, it also applies to users of the third-generation progestogens desogestrel and gestodene. However, the risk estimates for third-generation OCs was $82 \%$ and $86 \%$ higher than the risk estimates for OCs with LNG, a risk ratio even higher than for OCs with the fourth-generation drospirenone. That should not be the case, if the concern of S\&D had any substantial significance. The magnitude of misclassification of the short-term LNG users was in the order of 0.22 (per cent of short-term users) $\times 0.023$ (proportion of short-term users who were recorded within the first 3 months of 1995) $=0.005$ or about a half per cent. In addition, we stratified for (adjusted for) length of use when comparing the different types of progestogens, thereby eliminating all other differences (other than the small fraction of shortterm users starting their short-term use in 1995) concerning length of use between different OC types. Therefore, it is very unlikely that this small misclassification of short-term users starting their short-term use in the beginning of 1995 would have had a substantial influence on our estimates

Thereafter S\&D argue that the risk in shortterm users of OCs with LNG should have been three times higher than for long-term users. Our analysis demonstrated, however, that the risk among short-term users (all products considered together) was about $50 \%$ higher in the first year. Not more. With these national cohort data, their calculation, anticipating this three-fold difference, is far too high.

As indicated in our paper, ${ }^{2}$ a large number of studies with different designs have assessed a possible differential effect of different progestogens to influence women's VTE risk. The vast majority of these studies have found a consistently higher risk with OCs containing desogestrel and gestodene than for $\mathrm{OCs}$ containing LNG.

So the present two new studies are in accordance with the available scientific evidence. In addition, the different impact of the different progestogens on the so-called Activated Protein $\mathrm{C}$ sensitivity ratio gives us a probable mechanism through which these different progestogens exert their differential influence on the coagulation system.

In conclusion, well-sized and well-conducted newer epidemiological studies consistently find a higher risk of VTE with the newer progestogen types as compared with the older types. The fact that differently designed studies conducted at different times in different countries find the same differential risk between different progestogen types increases the probability that this difference is real and not due to bias and confounding as S\&D suggest.

Next S\&D argue that when operating with length of use one has to consider only the length of the last use. Had we done so, S\&D could have argued that our missing data on previous use had flawed our effort to exclude bias due to attrition of susceptible individuals, as this attrition is in effect according to the total length of use and not only according to the last length of use.

\section{Confounding}

Next S\&D argue that our missing control for obesity (BMI) "was a major defect in the Danish study". Now, adiposity is a well-established risk factor for VTE. A risk factor is, however, not the same as a confounder, which in addition to being a risk factor also has to be associated with use of OCs in general, and differentially with different OC types, if the considerations of S\&D are to be valid. The fact is that there is no association between OC use and adiposity, and no significant difference in the frequency of adiposity in users of different types of OC (as documented in our paper). ${ }^{2}$ Therefore, the increased risk of VTE in users of $\mathrm{OC}$ with third- and fourth-generation OCs as compared with OCs containing LNG cannot be explained by our missing control for adiposity.

Conversely, it is true that the frequency of adiposity increased in the general population during the study period. Therefore we adjusted our estimates for calendar year, thereby eliminating this potential time-trend bias.

S\&D further speculate that women at an increased risk of VTE should preferentially be prescribed newer OCs, in particular OCs containing drospirenone. Our data demonstrate the opposite. The use of medication for hypertension, diabetes, hyperlipidaemia and heart disease was actually lower in users of drospirenone than in users of LNG. Consequently, this speculation does not seem to be very relevant.

Finally, S\&D postulate that the decreasing risk of VTE with increasing length of education was unexpected, and therefore an indicator of selection bias, women educated for a short time being more prone to be diagnosed with VTE in case of symptoms than women with a longer education. This assumption is unlikely. All diseases I am aware of (with the one exception of multiple sclerosis), including thrombotic diseases, decrease in frequency with increasing length of education. Referral to hospital and subsequent diagnostic investigations are free in Denmark. Therefore, there is no reason to believe in any selection bias according to length of education. As our trend confirms a previously proven general trend towards unhealthier lifestyle and more morbidity with decreasing length of education, this finding only strengthens the validity of our results.

Other issues

S\&D postulate that the diagnoses in the National Register of Patients have not been validated. This 
is wrong. We went through all the VTE diagnoses during the period 1994-1998 in women aged 15-44 years and found 10\% with an uncertain diagnosis (this was clearly stated in the paper).

Initially, S\&D make a rather unusual complaint, namely that large observational studies nearly always find significant associations, even if the association is small. Is that really a critique? Meaning that if we had done a smaller study then the quality would improve? The quality in large samples is first of all that one is able to separate the contribution from different axes of OC use; the length of use, the estrogen dose, the progestogen type, the dose and the route of administration. Other scientists would consider this to be a strength rather than a weakness.

\section{Conclusion}

Scientific critique is always welcome, and bias and confounding in observational studies are difficult to exclude completely. Some of the suggestions made by $\mathrm{S} \& \mathrm{D}$ are theoretically valid but seem of little or no quantitative significance. When several large-scale, independent epidemiological studies generate the same results, one also has to consider the possibility that these results are actually true.

Øjvind Lidegaard, DrMSci

Professor, Rigshospitalet, University of

Copenhagen, Copenhaen, Denmark.

E-mail: Lidegaard@rh.regionh.dk

References

Shapiro S, Dinger J. Risk of venous thromboembolism among users of oral contraceptives: a review of two recently published studies. J Fam Plann Reprod Health Care 2010; 36: 33-38.

2 Lidegaard Ø, Løkkegaard E, Svendsen AL, Agger C. Hormonal contraception and risk of venous thromboembolism: national follow-up study. BMJ 2009; 339: b2890.

\section{Reply}

We thank Professor Lidegaard for his comments. ${ }^{1}$ As stated ${ }^{2}$ with regard to his study, ${ }^{3}$ "The investigators are to be congratulated for conducting such a large study that also adjusted for the confounder information assessable in the Danish registries". We also believe that additional subanalyses might improve the interpretability of the findings. We address Professor Lidegaard's comments in the paragraphs that follow.

\section{Previous studies}

Relevant references to studies of the risk of venous thromboembolism (VTE) in users of desogestrel and gestodene, as compared with levonorgestrel (LNG), were provided in our review, ${ }^{4}$ and a further detailed consideration of the "large number of studies" at issue would fall outside the scope of this response. Readers interested in the topic may also wish to refer to the judgement of the High Court of Justice ${ }^{5}$ in the UK. It is not primarily the court's conclusion that is of interest here but the comprehensive documentation of methodological details and arguments that were scrutinised during more than 12 weeks of hearings with expert witnesses on the strengths and weaknesses of all relevant studies on this topic published until 2002.

\section{The Danish cohort study}

Professor Lidegaard questions the relevance and quantitative impact of our criticisms. ${ }^{4}$ In response, we first consider the validity of the diagnosis of VTE in the Danish registry data.

\section{Validation}

In their publication, ${ }^{3}$ Lidegaard and his colleagues "clearly stated" that "the registry approach did not permit us to evaluate the validity of each included diagnosis of [VTE]" and that they relied on the "final discharge diagnosis as reported". The statement now made, that they "went through all the VTE diagnoses ... and found $10 \%$ with an uncertain diagnosis..."1 is misleading: that estimate was made in an earlier study. ${ }^{6}$ As indicated in our Letter to the Editor in this issue of the Journal, ${ }^{7}$ Severinsen and colleagues $^{8}$ have now reported that in Denmark registry-recorded diagnoses of VTE were incorrect in at least $40 \%$ of cases aged 50-64 years (in $40 \%$ the diagnosis could be ruled out, and in $5 \%$ it was uncertain) - or about $29 \%$ in female hospital-ward cases. It is unlikely that the discrepancy with the $10 \%$ rate of "uncertainty" identified by Lidegaard can be explained by age, and it obliges him to verify the diagnoses in his study. If VTE was incorrectly diagnosed as commonly as is suggested by Severinsen's data, the interpretation of the small risk estimates must be questioned.

The remainder of our response follows Lidegaard's sequence.

\section{Left censorship}

Lidegaard acknowledges that left censorship may have distorted the data for LNG more than for drospirenone. In fact, since drospirenone was only introduced in Denmark in 2001, for this compound there was no left censorship, and no distortion. Desogestrel and gestodene were introduced in Denmark in the first and second half of the 1980s, respectively, whereas LNG has been available since the first half of the 1970s. In addition, the market shares of desogestrel and gestodene were more or less stable between 1995 and 2005,9 while the use of LNG declined. Inevitably, therefore, the distorting effect of left censorship was more marked for LNG than for desogestrel or gestodene.

While Lidegaard acknowledges left censorship, his calculation of its potential impact is misleading for a number of reasons. Here however, we confine our response just to one of them. Lidegaard states that "about a half per cent" of "short-term levonorgestrel users" were misclassified. This misses the point. In the firs study year (1995) all long-term users (100\%) were misclassified as short-term users. As approximately $14 \%$ of the total LNG exposure stems from $1995^{9}$ it has to be assumed that in that year approximately 57000 woman-years of LNG use were classified as short-term use $(0.14 \times$ 411 099; see Table 2 in Lidegaard's publication) This number probably represents more than $60 \%$ of the total short-term exposures $(0.22 \times 411099$ Table 1). We therefore disagree with Lidegaard "that it is very unlikely" that this misclassification "had a substantial influence" on the risk estimates.

\section{Risk of VTE among short-term OC users}

As the duration of oral contraceptive (OC) use was misclassified, the risk of VTE for short-term users was underestimated in the Danish study. In an earlier study ${ }^{6}$ Lidegaard observed a three-fold higher relative risk increase for the first year of use relative to the following years. That study identified VTE from the same source (the Danish patient registry) but the information on OC use was derived from a different source: it was reported by the patients, and there was no left censorship. Moreover, it is not only short-term use that is at issue: in a valid comparison similar durations of use, whether short- or long-term, should have been compared among users of the different OCs.

\section{Total vs current duration of $\mathrm{OC}$ use}

With regard to the total duration of all episodes of OC use versus duration of the current episode only, we reiterate that multiple studies have demonstrated that the risk of VTE is no longer increased within a few months of stopping current use. It is only the duration of such use that is relevant. The need to have data on all episodes of OC use is not in order to sum all durations, but in order to be able to compare starters with starters, re-starters with re-starters, and switchers with switchers, as illustrated in Figure 1.

A comparison along these lines would also minimise any "bias due to attrition of susceptible individuals", 1 mentioned by Lidegaard. In addition, the requirement that starters should be compared with starters could readily have been met in the Danish study. Had follow-up commenced in 2001, the study would have started after the introduction of all the relevant progestogens and had women who used OCs between 1995 and 2000 been excluded, for practical purposes that objective would have been accomplished.

Confounding by obesity and other risk factors Lidegaard claims that he has documented that there was "no significant difference in the frequency of adiposity in users of different types of OC." In his study he had no data on obesity, ${ }^{3}$ and his claim is based on data from a different study covering the time from 1994 to $1998 .^{6}$ Those data do not preclude the possibility that preferential prescribing of selected OCs occurred after 1998, and there is evidence that it did occur. In a recent study, drospirenone - a progestogen that is also an aldosterone antagonist - was preferentially prescribed to women with a high body mass index (BMI). ${ }^{10}$ That study also demonstrated that the combination of obesity with other risk factors (e.g. family history) led to a multiplicative increase in the risk of VTE. Since the Danish study lacked data on BMI, confounding from that source was not ruled out. Lidegaard acknowledges the increase in the prevalence of obesity that occurred between 1995 and $2005,1,11$ as well as the decline in the use of LNG. ${ }^{9} \mathrm{He}$ nevertheless claims that adjustment for calendar year eliminated confounding due to obesity. Both the increase in obesity and the decline in the use of LNG were substantial. Thus

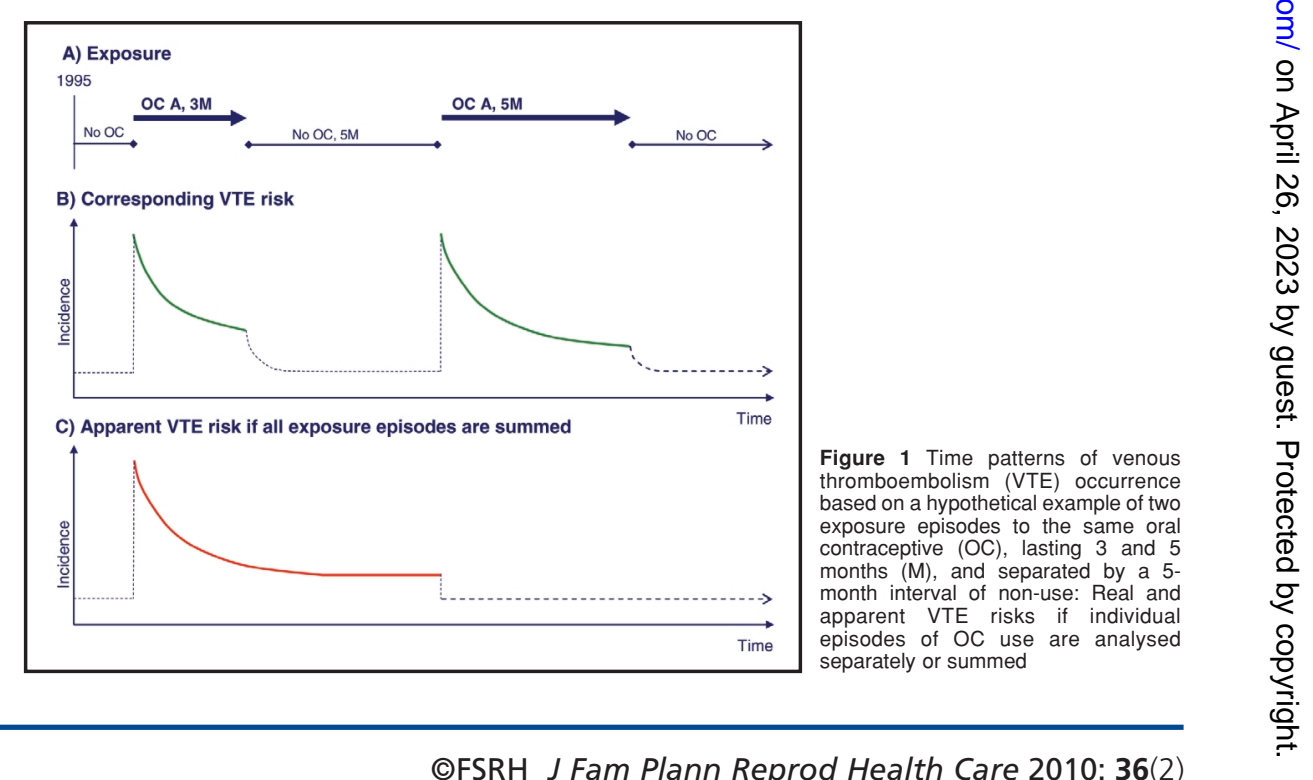

